\title{
Editorial
}

\section{Current concepts in the articular cartilage repair and regeneration}

\section{Introduction}

Hyaline cartilage provides a smooth articular surface and the ability to withstand good amount of pressure. It is alymphatic, aneural, avascular loadbearing tissue, composed of chondrocytes sparsely embedded within an extracellular matrix of collagens and proteoglycans. Articular cartilage damage has inherent limited healing potential, hence it remain as a challenging problem for orthopedic surgeons. In the immediate past, surgeons often replaced the articular surface with implants when articular lesions become full-blown osteoarthritis. Recently there are new orthobiological techniques in cartilage lesions with commendable effectiveness to regenerate tissue homeostasis and delay the progression of osteoarthritis. Due to hypocellularity and avascularity, articular cartilage has limited capacity for regeneration upon injury. Articular cartilage injuries are commonly caused by sports and recreational activities. When left untreated, articular cartilage lesions can lead to osteoarthritis

Current treatment modalities for articular cartilage repair help to repair the articular cartilage lesions and reduce pain in affected joints to some degree.

Since these techniques often generate inferior fibrocartilage repair and has issues of donor-site morbidity which necessitates the search for an alternative best method. Mesenchymal stem cells (MSCs), have emerged as a promising cell source for cartilage repair. Stem cells may be delivered directly by means of injection or seeded in scaffolds for implantation. Major advantages of applying mesenchymal stem cells for cartilage repair are from their easy availability, multilineage potency and proliferative capacity.

The most modern trend includes preventive interventions and therapeutic solutions that can promote the tissue regeneration and the reduction of cartilage degeneration.

\section{The facts}

Damaged articular cartilage has poor capacity for self-repair. The self repair depends on the size, depth and location of the defect, apart from the age of the patient. ${ }^{1,2}$ Cartilage defects are classified in to two types according to the depth of the lesion in to partial (chondral) or full thickness (osteochondral).

The healing of chondral defects do not by the host blood supply, resident macrophages and MSCs originating from the bone marrow because it not penetrate the vascularised subchondral bone. Instead the repair mostly relies on the limited mitotic activities of resident chondrocytes which are rarely effective. On the other hand, the full thickness (osteochondral) defects are lesions that penetrate the subchondral bone, and in such cases, the bone marrow provides vascularisation and MSCs to promote the better repair. ${ }^{3}$

Articular cartilage lesions, when left untreated, forms fibrocartilage, leading to the very early onset of degenerative osteoarthritis. ${ }^{4,5}$ The poor intrinsic regenerative capacity of articular cartilage demands the importance of an effective treatment method for cartilage repair to avoid this complication. Nonsurgical treatment of cartilage lesions has demonstrated variable results. A number of surgical procedures like marrow stimulation techniques or microfracture, osteochondral autografting/allografting and cell-based therapies have been successfully used clinically. ${ }^{6}$

\section{Current treatment modalities}

Microfracture is a common procedure in which small holes are made and distributed across the entire articular cartilage lesion site, at a distance of 3-4 mm apart and down to a depth of $4 \mathrm{~mm}$, thus yielding about 3-4 holes per $\mathrm{cm} 2 .^{7-9}$ It is a minimally invasive arthroscopic approach, which does not require any costly instrumentation.The microfracture is a marrow stimulation techniques which brings the marrow cells to the defect site to help in repair. ${ }^{10}$

Mosaicplasty/Osteochondral Grafting was introduced in the early 1990s. ${ }^{11}$ Here the osteochondral plugs are taken by a cylindrical cutting device from healthy cartilaginous area in the form of cylindrical shaped plugs, and then implanted into an articular cartilage defect. The osteochondral plugs are usually taken from the non-weight bearing peripheries of both femoral condyles near the patellofemoral joint and introduced as a mosaic to fit into the cartilage defect.

Autologous Chondrocyte Implantation (ACI) is a cell-based technique to treat the full-thickness chondral defects in the knee. ${ }^{12}$ Here the cartilage tissue is first harvested from the patient by arthroscopy from a non-weight bearing area. Then the chondrocytes are isolated and culture in the laboratory to form a monolayer culture to get the desired population of chondrocytes. Thereafter, they are transplanted into the cartilage defect and held in place by sewing a periosteum patch over it so as to localise the chondrocytes within the defect site. ${ }^{13}$

Stem Cells for Articular Cartilage Repair and Regeneration has recently emerged as a promising option to treat articular defects and early OA stages. There are mesenchymal stem cells and pluripotent stem cells used in these group. 
Mesenchymal Stem Cells (MSCs) are the most widely used stem cell source for articular cartilage regeneration technique. It can be easily derived from a wide variety of tissues including the bone marrow, muscle, blood, synovium and synovial fluid. ${ }^{14-18}$

These cells are easy to expanded to large numbers and display a strong ability to differentiate to multiple lineages including chondrogenic, osteogenic and adipogenic. ${ }^{3}$ Among the MSCs, synovium-derived MSCs have been reported to have superior proliferation and differentiation capacity into chondrocytes, supporting their use in cartilage tissue engineering and regeneration. $^{19}$

Human pluripotent stem cells has the great potential of selfrenewal and ability of differentiation to cell lineages of all three germ layers. $^{20}$ The embryonic stem cells (ESCs) (Thomson et al., 1998) and induced pluripotent stem cells (iPSCs) (Takahashi et al., 2007) are the two major sources of pluripotent stem cells. The embryonic stem cells are isolated from the inner cell mass of embryos, where as pluripotent stem cells are created from both foetal and adult somatic cells. Pluripotent stem cells are not suitable for direct clinical application ${ }^{21}$ because of tumorigenic property, therefore, chondrogenic differentiation of pluripotent stem cells to desired purified populations of chondroprogenitors or chondrocytes has to be done prior to transplantation. This can be achieved by culture in defined media supplemented with growth factors and coculturing with chondrocytes. ${ }^{22}$

Stem cell behaviours are regulated by multiple microenvironmental controls. As an external signal, mechanical stiffness of the extracellular matrix is capable of governing stem cell fate determination.

In order to deliver stem cells for articular cartilage regeneration, an appropriate scaffold is important. Biomaterials that possess the characteristics such as biocompatibility (to support the viability, expansion and differentiation of seeded cells), biodegradability (to facilitate tissue remodelling with neotissue formation and matrix deposition) and three-dimensional (3-D) structure are preferred for the scaffold.Two materials have been used as scaffolds for cartilage tissue engineering. One is naturally derived biopolymers such as alginate, hyaluronic acid ${ }^{23,24}$; Hyaluronic acid has emerged as the promising material for cartilage tissue engineering from the natural biopolymers. ${ }^{24}$

Polylactic acid (PLA), poly(lactic/glycolic acid) (PLGA) and polyglycolic acid (PGA) are the synthetic polymers that have also been used commonly.

Bioactive factors are another promising factors used in articular cartilage regeneration are transforming growth factor- $\beta s$ (TGF- $\beta s$ ), insulin-like growth factor-I (IGF-I), fi broblast growth factor (FGF) and bone morphogenetic proteins (BMPs). Among BMPs, BMP-2 stimulates the proteoglycan synthesis and improve the repair of osteochondral defects, ${ }^{25}$ Similarly the combination of TGF- $\beta$ s and BMPs has also been demonstrated to be effective in enhancing MSC chondrogenesis.

Oxygen Tension is the next external factor influencing the Stem cell behaviours. The ideal physiological environment of articular cartilage is in low oxygen. It has been shown that hypoxic culture condition ( $5 \% \circ 2$ ), enhances the matrix synthesis of chondrocytes with the expression of type II collagen, but declined when 02 levels fell below $1 \%{ }^{26}$ Oxygen tension also modulate stem cell chondrogenesis and exhibits significant effects on the metabolism of articular cartilage, including changes in synthesis of glycosaminoglycans (GAGs) and secretion of growth factors by chondrocytes. $^{26}$

Mechanical Forces like motion and appropriate loading of synovial joints are necessary for the proper structure, function and metabolism of the articular hyaline cartilage. Numerous reports have linked that the mechanical stress is an important modulator of the native articular cartilage metabolic activities and to serves to maintain the cartilage homeostasis. ${ }^{27}$ Where as excessive mechanical forces may lead to cartilage damage and development of osteoarthritis as well. ${ }^{27}$

\section{Discussion}

Articular cartilage repair and regeneration continue to be challenging because of the poor regenerative property. Various cartilage modalities have described with great potential for improving articular cartilage therapy. More recently, a variety of promising cell sources, biocompatible tissue engineered scaffolds, scaffoldless techniques, growth factors, and mechanical stimuli used in current articular cartilage tissue engineering have also evolved. The role of tissue engineering in articular cartilage repair and regeneration is promising. In the pre-autologous chondrocyte transplantation Era, the British anatomist Hunter states "Cartilage injury is a troublesome thing and once injured is seldom repaired" was the general axiom for thinking about cartilage repair.

Studies have shown that microfracture techniques do not fill in the chondral defect fully, and it forms fibro cartilage rather than hyaline cartilage. The microfracture techniques became controversial due to a lack of favourable reports on the long-term effects.

In mosaicplasty the defects can be filled immediately with mature, hyaline articular cartilage is the main advantage, moreover both chondral and osteochondral defects can be treated in the same way. This procedure can be performed either as an open or arthroscopically assisted procedure

The clinical outcomes of ACI therapy are truly encouraging. It has signifi cantly reduced pain and more interestingly the formation of a hyaline-like cartilage being observed.

$\mathrm{ACI}$ is a two-step procedure in which the new cartilage cells are grown and then implanted in the cartilage defect. ACI is most useful for younger patients who have single defects larger than $2 \mathrm{~cm}$ in diameter. ACI has the advantage of using the patient's own cells, and it does have the disadvantage of being a two-stage procedure that requires an open incision.

The ACI technique has been proven to be a promising treatment modality for treatment of cartilage lesions and which has resulted in good clinical success. ${ }^{28}$ Way back in 1980 s, it is well understood that the concept of healing cartilage with predominantly hyaline tissue is a myth. Then it was hypothesized that hyaline cartilage repair could be achieved by a cell-based approach to the problem. $^{29}$ This thinking helps to develop a new method for achieving the goal of hyaline cartilage repair.

The clinical application of MSCs for the treatment of articular cartilage defects and OA shows promising results. ${ }^{30}$ MSCs are a good candidate for cell therapies and their healing potential has been explored also in terms of cartilage regeneration. ${ }^{31}$ The use of MSCs in the clinical setting though can be considered safe with good clinical improvement, positive MRI and macroscopic findings, but histologic features gave more controversial. Similarly even though numerous advancements have been made, the understanding of MSCs mechanism of action as well as their potential and limitations for the clinical use remain controversial. ${ }^{32}$ Use of scaffolds coated to chemotactically enhance mesenchymal stem cell recruitment to the repair construct is an attractive option. There is a new concept emerging suggesting the need to treat the whole joint as an organ system and not just the cartilage lesion.

\section{Conclusions}

A biological approach to cartilage lesions is a new challenge since it has inherent limited healing potential. A number of viable options have been made available over the years to address these problems. Every technique has its own merits and demerits. Stem 
cells therapy is a strong promise in the treatment of cartilage defects and osteoarthritis.

Stem cells, in particular MSCs, are expected to revolutionise the treatment for cartilage defects and osteoarthritis in the near future. The future of the field is now looking to undertake the repair of whole cartilage surfaces beyond focal defects.

\section{References}

1. Mobasheri A, et al. Mesenchymal stem cells in connective tissue engineering and regenerative medicine: applications in cartilage repair and osteoarthritis therapy. Histol Histopathol. 2009;24(3):347-366.

2. Convery FR, Akeson WH, Keown GH. The repair of large osteochondral defects an experimental study in horses. Clin Orthop Relat Res. 1972;82:253-262.

3. Toh WS, et al. Advances in mesenchymal stem cell-based strategies for cartilage repair and regeneration. Stem Cell Rev. 2014;10(5):686-696.

4. Buckwalter JA, Rosenberg L, Hunziker E. Articular cartilage: composition, structure, response to injury, and methods of facilitating repair. In: Ewing J, ed. Articular Cartilage and Knee Joint Function: Basic Science and Arthroscopy. New York: Raven Press Ltd.; 1990:19-56.

5. Mow V, et al. Experimental studies on repair of large osteochondral defects at a high weight bearing area of the knee joint: a tissue engineering study. $J$ Biomech Eng. 1991;113(2):198-207.

6. Redman SN, Oldfi eld SF, Archer CW. Current strategies for articular cartilage repair. Eur Cell Mater. 2005;9(23-32):23-32.

7. Steadman J, Rodkey W, Briggs K. Microfracture to treat full-thickness chondral defects: surgical technique, rehabilitation, and outcomes. J Knee Surg. 2001;15 (3): $170-176$.

8. Steadman JR, et al. Outcomes of microfracture for traumatic chondral defects of the knee: average 11-year follow-up. Arthroscopy. 2003;19(5):477-484.

9. Miller BS, et al. Patient satisfaction and outcome after microfracture of the degenerative knee. J Knee Surg. 2004;17(1):13-17.

10. Steadman JR, Rodkey WG, Rodrigo JJ. Microfracture surgical technique and rehabilitation to treat chondral defects. Clin Orthop Relat Res. 2001;391:S3629.

11. Hangody L, Berta A. Surgical techniques in cartilage repair surgery: osteochondral autograft transfer (OATS, Mosaicplasty). In: Shetty AA, ed. et al. Techniques in Cartilage Repair Surgery. Berlin Heidelberg: Springer; 2014:131-140.

12. Brittberg M, et al. Treatment of deep cartilage defects in the knee with autologous chondrocyte transplantation. New Engl J Med. 1994;331(14):889895.

13. Micheli LJ, et al. Autologous chondrocyte implantation of the knee: multicenter experience and minimum 3-year follow-up. Clin J Sport Med. 2001;11(4):223-228.

14. Pittenger MF, et al. Multilineage potential of adult human mesenchymal stem cells. Science. 1999;284(5411):143-147.

15. Toh WS, et al. Combined effects of TGFß1 and BMP2 in serum-free chondrogenic differentiation of mesenchymal stem cells induced hyalinelike cartilage formation. Growth Factors. 2005;23(4):313-321.
16. Fu WL, Zhou CY, Yu JK. A new source of mesenchymal stem cells for articular cartilage repair MSCs derived from mobilized peripheral blood share similar biological characteristics in vitro and chondrogenesis in vivo as MSCs from bone marrow in a Rabbit model. Am J Sports Med. 2014;42(3):592-601.

17. De Bari C, et al. Multipotent mesenchymal stem cells from adult human synovial membrane. Arthritis Rheum. 2001;44(8):1928-1942.

18. Ando W, et al. Clonal analysis of synovial fl uid stem cells to characterize and identify stable mesenchymal stromal cell/mesenchymal progenitor cell phenotypes in a porcine model: a cell source with enhanced commitment to the chondrogenic lineage. Cytotherapy. 2014;16(6):776-788.

19. Vinardell T, et al. A comparison of the functionality and in vivo phenotypic stability of cartilaginoustissues engineered from different stem cell sources. Tissue Eng Part A. 2012;18(11-12):1161-1170.

20. Thomson JA, et al. Embryonic stem cell lines derived from human blastocysts. Science. 1998;282(5391):1145-1147.

21. Heng BC, Cao T, Lee EH. Directing stem cell differentiation into the chondrogenic lineage in vitro. Stem Cells. 2004;22(7):1152-1167.

22. Toh WS, et al. Effects of culture conditions and bone morphogenetic protein 2 on extent of chondrogenesis from human embryonic stem cells. Stem Cells. 2007;25(4):950-960.

23. Toh WS, Cao T. Derivation of chondrogenic cells from human embryonic stem cells for cartilage tissue engineering. Methods Mol Biol. 2016;1307:26327910.1007/7651_2014_89.

24. Kim IL, Mauck RL, Burdick JA. Hydrogel design for cartilage tissue engineering: a case study with hyaluronic acid. Biomaterials. 2011;32(34):8771-8782.

25. Pecina M, et al. Articular cartilage repair: the role of bone morphogenetic proteins. Int Orthop. 2002;26(3):131-136.

26. Fermor B, et al. Oxygen, nitric oxide and articular cartilage. Eur Cell Mater. 2007;13(11):56-65.

27. Arokoski JP, et al. Normal and pathological adaptations of articular cartilage to joint loading. Scand J Med Sci Sports. 2000;10(4):186-198.

28. Grande Daniel A, Schwartz John A, Brandel Eric. Nadeen O. chahine articular cartilage repair where we have been, where we are now, and where we are headed. Cartilage. 2013;4(October (4)):281-285.

29. Sokoloff L, Edward A. Dunlop lecture: cell biology and the repair of articular cartilage. J Rheumatol. 1974;1(1):9-16.

30. Kon E, Filardo G, Roffi A, Andriolo L, Marcacci M. New trends for knee cartilage regeneration: from cell-free scaffolds to mesenchymal stem cells. Curr Rev Musculoskelet Med. 2012;5:236-243.

31. Filardo G, Madry H, Jelic M, Roffi A, Cucchiarini M, Kon E. Mesenchymal stem cells for the treatment of cartilage lesions: from preclinical findings to clinical application in orthopaedics. Knee Surg Sports Traumatol Arthrosc. 2013;21:1717-1729 [m].

32. Filardo Giuseppe, Perdisa Francesco, Roffi Alice. Maurilio Stem cells in articular cartilage regeneration. J Orthopaedic Surg Res. 2016;201611:42.

Dr. Raju Karuppal

Additional Professor, Department of Orthopaedics, Government Medical College Kozhikode, Kerala 673008, India

E-mail address: drrajuortho@rediffmail.com (R. Karuppal). 